\title{
Non-lieux et déterritorialisation, tropicalisation et reterritorialisation dans Passages d'Émile Ollivier et Chronique de la dérive douce de Dany Laferrière.
}

\author{
JOUBERT SATYRE
}

Université de Guelph

Résumé

Dans les littératures migrantes, l'espace est souvent un lieu de passage, hostile et vide de toute dimension humaine. Pour (re)trouver ses repères, le personnage migrant doit reconfigurer ce « non-lieu » qu'est le pays d'accueil, au risque d'en faire le terrain d'un conflit irrésolu entre deux cultures. Parfois, l'espace de la migration se mue alors en théâtre de l'entre-deux. Cet article tente d'analyser les processus de reterritorialisation de l'espace de la migration dans Passages d'Émile Ollivier et Chronique de la dérive douce de Dany Laferrière, en partant de l'hypothèse qu'il y a des liens entre non-lieux et déterritorialisation ainsi qu'entre tropicalisation et reterritorialisation.

\section{Introduction}

Dans les littératures migrantes, l'espace prend des formes et des sens divers. Espace dysphorique, locus horribilis, pour le personnage migrant, il se révèle comme un lieu de passage, lieu anonyme, vide de toute dimension humaine. Mais l'espace est aussi objet et lieu de rite d'initiation, de rite de passage. D'une façon ou d'une autre, c'est à travers l'espace que le personnage migrant fait d'abord I'expérience de l'altérité : il y trouve des êtres, des objets, des coutumes, bref un système symbolique dont il ne possède ni la clef ni l'usage. L'espace du pays d'accueil est, d'une certaine manière, constitué d'un ensemble de non-lieux, pour reprendre le terme de Marc Augé, non-lieux qu'on peut rapprocher de la déterritorialisation de Deleuze/Guattari. Les non-lieux sont des lieux de passage, lieux qui déshumanisent et où il est impossible de vivre, car contrairement aux lieux anthropologiques qui, selon Marc Augé, « créent du social organique » (Augé 119), les non-lieux « créent de la contractualité solitaire »(Ibid.). Dès lors, s'il veut survivre, le migrant doit trouver des stratégies pour habiter les non-lieux de la migration, autrement dit, passer de la déterritorialisation à la reterritorialisation', se fixer quelque part dans des lieux auxquels il donnera lui-même une dimension humaine, à la mesure de sa culture, de ses valeurs : il doit devenir chtonien, en quelque sorte. L'espace n'acquiert du sens pour le migrant que s'il est reterritorialisé. Mais peut-il l'être complètement puisque le migrant ne trouve jamais un espace vide? L'espace de la migration peut être alors vu comme un lieu agonistique où s'affrontent le même et l'autre, I'autochtone et l'allochtone. C'est un espace de l'entre-deux : un espace tropicalisé, pour reprendre le terme de Kundera. L'incapacité à habiter l'espace de la migration débouche sur la folie, le suicide ou le retour fantasmé au pays natal. Dans mon article, je tenterai de montrer, d'une part, les rapports entre déterritorialisation et non-lieux, d'autre part, entre reterritorialisation et tropicalisation dans deux textes 
ayant des éléments autobiographiquesii : Chronique de la dérive douce de Dany Laferrière et Passages d’Émile Ollivier.

\section{Non-lieux, déterritorialisation}

Selon Marc Augé, « si un lieu peut se définir comme identitaire, relationnel et historique, un espace qui ne peut se définir ni comme identitaire, ni comme relationnel, ni comme historique définira un non-lieu » (Augé 100). Il énumère l'ensemble de ces non-lieux qui caractérisent ce qu'il appelle la surmodernité : «Un monde où I'on naît en clinique et où I'on meurt à I'hôpital, où se multiplient, en des modalités luxueuses ou inhumaines, les points de transit et les occupations provisoires (les chaînes d'hôtels et les squats, les clubs de vacances, les camps de réfugiés, les bidonvilles promis à la casse ou la pérennité pourrissante) [...] » (Ibid.). À cette liste, il ajoute les voies aériennes, ferroviaires, autoroutières et les habitacles dits moyens de transport (avions, trains, cars), les aéroports, les gares, les parcs de loisir, les grandes surfaces de distribution. Quelques-uns de ces non-lieux sont omniprésents dans les deux textes étudiés. Ces non-lieux semblent des figures de la déterritorialisation et de l'errance du personnage migrant qui n'a pas encore trouvé sa place ou soleil ou bien qui ne la trouvera jamais. Désormais incontournable dans le champ des études francophones, la notion de déterritorialisation « indique un sentiment de perte ou de dépossession »(Bertrand 2005). En tête de ces non-lieux figure l'aéroport, lieu de passage, de l'éphémère, par où transitent des millions de destins qui ne se croisent que rarement, ce qui illustre l'un des paradoxes de la modernité exprimé dans ce cri de Baudelaire: « Multitude, solitude $[\ldots] »$ («Les Foules », Petits Poèmes en prose).

Cependant loin d'être uniquement un lieu de l'anonymat et du passage, l'aéroport semble également un lieu d'initiation. C'est dans un aéroport que le narrateur de Chronique de la dérive douce fait sa première expérience de l'altérité du pays d'accueil : « Un couple en train de s'embrasser à l'aéroport. Un baiser interminable. La fille est en minijupe rouge. Cette scène aurait provoqué une émeute à Port-auPrince. Ici, les gens passent sans même y jeter un œil. Je suis le seul à m'intéresser à ce baiser rouge » (Laferrière 11-12). Le narrateur, étonné, ne peut s'empêcher de comparer sa culture à celle qu'il vient à peine de découvrir afin d'en faire ressortir les différences. D’ailleurs, à la fin du récit, la même scène vue à quelques pas de lui n'attire guère son regard : « À moins d'un mètre de moi. Un long baiser passionné. La fille est en minijupe rouge. Je passe sans m'arrêter » (Laferrière 129). On comprend qu'il aura fait son apprentissage, après un an sur la terre d'accueil. Dans le récit de Laferrière, l'aéroport est donc présent, comme il se doit dans la plupart des écrits sur la migration, mais tout en étant un non-lieu, il est une 
possibilité d'initiation pour le personnage migrant. Il est seuil, porte d'entrée dans un monde nouveau dont il laisse entrevoir le potentiel de désorientation mais aussi d'aventures.

Le mot aéroport est plusieurs fois employé dans Passages : « Au mitan de cette chaude journée de janvier, Normand Malavy débarque à I'aéroport de Miami. Après avoir longé les interminables couloirs de terrazzo, il se retrouve devant le comptoir de contrôle des papiers d'identité » (Ollivier 67). Ailleurs dans le roman, c'est I'aéroport de Maïs Gâté de Port-au-Prince qui est évoqué, au moment du départ de Normand pour l'exil. À ces aéroports qui sont nommés, il faut en ajouter d'autres que le roman passe sous silence ou du moins qu'il laisse deviner. On peut citer celui de Montréal, où Normand a atterri au terme de son voyage d'exil, et d'où il est parti pour se rendre à Miami. Il y a aussi l'aéroport de La Havane où a débarqué la Cubaine Amparo lors de son retour au pays natal. L'aéroport joue pleinement ici son rôle de non-lieu : il est seulement un espace de passage et de transition L'autre non-lieu de Passages est le camp Krome où croupissent les boat people qui ont fait naufrage en tentant de gagner Miami. Ce camp est le prolongement terrestre de I'océan qui a englouti la majeure partie d'entre eux. Ces deux non-lieux sont des symboles de déracinement, donc de mort. On ne peut pas y vivre. Voilà pourquoi l'une des rescapés, Brigitte Kadmon, qui s'y trouve enfermée, décide de retourner au pays natal, bien que Normand lui offre de l'aider pour s'installer aux États-Unis :

Je retourne à Port-à-l'Écu. Je veux vivre, prier, être enterrée dans ma langue. Ici, sous les reflets des néons, à l'ombre des gratte-ciel de béton, d'acier et de verre, les gens ont quelque chose de triste qui laisse l'impression qu'ils sont au terme de leur vie. Là-bas, face à la mer, à marcher contre le vent, contre les brisants, on ressent un élan de vie, un désir de lutter, de vaincre [...]. (Ollivier 229-230)

Vivre dans des non-lieux, c'est comme être au seuil de la mort. Ceux qui n'arrivent pas à en sortir n'ont d'autres choix que la mort ou le retour à la terre natale. Être enterré dans sa langue, comme le souhaite Brigitte Kadmon, n'est-ce pas le désir de retrouver sa terre natale, ce lieu anthropologique par excellence qu'on n'a pas besoin de connaître, puisqu'il est la substance même de notre vie, mais de reconnaître? La perte de la terre natale est aussi celle de la langue maternelle, car il est rare que les visages et les paysages de l'exil parlent notre langue. En ce sens, la langue maternelle est aussi un lieu que nous habitons dès l'enfance; sa perte nous livre aux vertiges du non-lieu. Mais il s'agit ici d'un lieu psychique, selon les termes de Simon Hareliii. De ce point de vue, toute langue étrangère que nous ne maîtrisons pas peut être considérée comme un non-lieu.

D’autres non-lieux sont présentés dans Chronique de la dérive douce. Ils marquent les différentes haltes dans l'errance du narrateur qui n'a pas encore trouvé un endroit où se fixer. Parmi ces non-lieux qui expriment la déterritorialisation du narrateur, on peut citer le parc. C'est un non-lieu parce que le protagoniste n'en connaît pas I'histoire, mais surtout parce que c'est un espace vide où il n'a que des 
pigeons comme compagnons. Les nombreuses rues (Sherbrooke, Saint-Denis, Sainte-Catherine) où le narrateur se promène, la gare, le métro, l'usine sont les différentes étapes de cette cartographie de l'errance qu'est Chronique de la dérive douce; ces non-lieux sont les points de chute du narrateur dans sa découverte de la topographie de Montréal. Il est à souligner que c'est dans ces non-lieux que le narrateur rencontre ses premiers amis : le clochard, I'Africain, I'Indien, qui appartiennent tous à une communauté déracinée, déterritorialisée comme lui. Mais comme l'aéroport, ils ont une valeur initiatique pour le protagoniste. À force d'être fréquentés, ils transcendent leur fonction première pour devenir presque des rituels.

Le temps semble aboli pendant ces errances où le narrateur fait son apprentissage de la ville (le verbe apprendre est plusieurs fois employé). Il est aboli parce que le narrateur a perdu ses repères culturels, familiaux, sociaux. Le pays d'accueil est donc un immense non-lieu, une sorte de labyrinthe à ciel ouvert dans lequel on risque de se perdre. Seul compte alors l'exploration de l'espace, qui permettra de le baliser, d'y tracer des trajectoires, d'y vivre, puisque le protagoniste déclare qu'il n'est « pas un touriste de passage »(14). Toute I'activité du narrateur semble se réduire à cette exploration. La surabondance des verbes qui appartiennent au champ lexical du déplacement souligne cette obsession de l'espace: « Je marche, je marche, je marche toute la nuit dans la nouvelle ville » (Laferrière 18 ) ; « Je passe devant ce magasin »(25); «Je tourne au coin de la rue » (26). Tous ces verbes soulignent les déambulations d'un corps qui est dérouté, déterritorialisé, qui n’a pas encore de lieu fixe mais qui veut aussi découvrir le pays d'accueil. Ces pérégrinations sont aussi des rites d'initiation. Tous ces non-lieux finissent par perdre leur étrangeté pour s'inscrire dans les paysages familiers, quotidiens du narrateur.

À cette étape de l'exil, le pays natal n'est pas trop loin, il est à fleur de mémoire et pour l'évoquer, l'invoquer, il suffit de fermer les yeux. Le personnage migrant semble alors vivre dans un entredeux, un écartèlement schizophrénique; non pas qu'il possède une double identité, mais parce qu'il y a scission entre l'espace où est son corps et celui où se trouve son être psychique, sa mémoire. Celui-là est ici; celui-ci, ailleurs, un ailleurs temporel, on en convient, mais aussi spatial. Cette scission est maintes fois rappelée dans Chronique de la dérive douce. Il suffit de fermer les yeux pour rétablir la relation mémorielle avec la terre natale, grâce à laquelle les souvenirs surgissent, souvenirs qui conjurent les malheurs du présent, pour chimérique que soit cette résurgence. Fermer les yeux, c'est s'arracher mentalement au monde présent, à l'espace dans lequel on se trouve pour qu'émerge le monde de la mémoire. Le narrateur est encore en pleine déterritorialisation. Tout autour de lui est étrange, insolite. Il ne peut encore habiter pleinement l'espace de la migration ni retourner en terre natale non plus. La convocation du passé permet de superposer deux mondes : I'un mental et psychique; I'autre concret et 
physique. Le corps n'est pas encore tout à fait d'ici, mais il n'est plus d'ailleurs :

« Certaines fois, je n'ai qu'à fermer les yeux pour me croire là-bas. Le bruit des voitures est partout le même » (17). Assez souvent, le narrateur croit retrouver dans une scène du pays d'accueil les réalités de la terre natale. Ainsi, pendant un marathon, les clameurs d'encouragement de la foule lui parviennent comme un chant créole : «Le soleil me frappe de plein fouet. Pendant quelques secondes, j'ai cru que j'étais à Port-au-Prince et que je descendais le morne Nelhio vers le stade Sylvio Cator. Les voix des gens massés le long de la rue Sherbrooke, encourageant les coureurs du marathon, me parviennent comme un chant créole » (21). Les événements présents sont interprétés en fonction de ceux du pays natal, qui leur donnent sens. Passé et présent s'inscrivent dans un continuum qui permet au migrant de s'adapter à son nouvel environnement.

\section{Tropicalisation, reterritorialisation}

Comment le personnage migrant arrive-t-il à reterritorialiser l'espace de la migration, autrement dit à transformer les non-lieux en des lieux où il peut retrouver ses repères, des lieux de relation et d'identité, ou pour citer Jean-Pierre Bertrand, compenser « le sentiment de dépossession » de la déterritorialisation par celui de l'identité retrouvée? Pour paraphraser Appadurai, comme les rites de passages qui inscrivent les lieux dans un corps, le personnage migrant doit, à sa manière, inscrire son être, son corps dans l'espace de la migration, s'il ne veut pas vivre dans la mélancolie, dans le désir inassouvi du retour au pays natal.

L'une des stratégies employées par le personnage migrant pour habiter l'espace du pays d'accueil est ce que Kundera appelle la tropicalisation. Il crée ainsi un espace-refuge, un ethnoscape, selon Appadurai. Bien qu'il n'ait pas défini ce qu'il entend par tropicalisation, Kundera la considère comme un trait « du roman d'au-dessous du trente-cinquième parallèle ou [...] roman du Sud : une nouvelle grande culture romanesque caractérisée par un extraordinaire sens du réel lié à une imagination débridée qui franchit toutes les règles de la vraisemblance »(42). Le mot tropicalisation est employé de manière spécifique à propos des Versets Sataniques de Rushdie :

Farishta vole au-dessus de Londres et désire «tropicaliser » cette ville hostile : il résume les bénéfices de la tropicalisation : « I'institution d'une sieste nationale [...] de nouvelles variétés d'oiseaux sur les arbres (aras, paons, cacatoès), de nouvelles espèces d'arbres sous les oiseaux (cocotiers, tamariniers, banians barbus) [...] ferveur religieuse, agitation politique [...] les amis qui débarquent les uns chez les autres sans prévenir, fermeture des maisons de retraite, importance des grandes familles, nourriture plus épicée [...]. Désavantages : choléra, typhoïde, maladie du légionnaire, cafards, poussière, bruit, une culture de l'excès. » (43)

http://www.brocku.ca/cfra/voixplurielles05-02/index.html 
À partir de cet extrait, on peut définir la tropicalisation comme une forme de reterritorialisation : le migrant transplante dans la terre d'accueil sous le mode fantasmé ou réaliste les éléments de sa culture, créant ainsi un espace d'hybridité, selon le terme d'Homi Bhabha.

Cette tropicalisation de l'espace est clairement exprimée dans Passages d'Émile Ollivier. Leyda, la veuve de Normand Malavy, traverse le quartier Notre-Dame-de-Grâce pour se rendre au marché du coin. Le lecteur découvre ce quartier à travers son regard et par la technique de la description-promenade qui est un balisage de l'espace. Leyda arrive dans un parc qui, selon le narrateur, « avait été le théâtre de conflits violents entre des jeunes Noirs qui habitaient la plupart en contrebas de la rue Sherbrooke, au sud, et des adolescents juifs logés en majorité au nord. Depuis, il est devenu un lieu frontière, un point de démarcation, une ligne de fracture entre deux solitudes » (Ollivier 38). L'espace est ici délimité, marqué. Fait intéressant à noter : entre les quartiers des jeunes de deux communautés diasporiques, c'est un non-lieu, un parc, symbole d'errance et de déterritorialisation, qui sert de ligne de démarcation. Le narrateur nous apprend que « le quartier avait résonné de rythmes endiablés, de spectacles insolites, de liesses souveraines » (Ibid.), expressions qui décrivent le carnaval antillais.

Alors que la tropicalisation dans Versets Sataniques reste au niveau du désir, du fantasme, elle est bien réelle dans Passages, au moins le temps du carnaval antillais. En effet, le narrateur parle de « toutes les couleurs de peaux se côtoyant dans une débauche de costumes bigarrés, une foule riant haut et fort, une horde de corps que des coulées de sueurs font luire au soleil; cette partie de la ville soudain devenue folle »(Ibid.). L'espace tropicalisé est modifié, formaté, pourrait-on dire, pour accueillir ces corps autres qui I'ont ainsi dépouillé de son autochtonie pour le rendre autre, allochtone. Voilà pourquoi le narrateur a pu noter : « cette partie de la ville devenue soudain folle. »

La tropicalisation est vue comme un coup de force contre l'espace du pays d'accueil, contre la raison et tous ses corollaires : la pudeur, l'ordre, le principe de réalité : « Et I'on voit passer des bipèdes obscènes sur des plates-formes mobiles, défilés de couples mimant des scènes d'accouplement [...] masques, rubans de dentelles, serpentins qui deviennent cerceaux emmêlés, démêlés, cercles de femmes, femmes-libellules, femmes-tortues, femmes-lézards » (Ollivier 38-39). L'hybridité (dans son sens étymologique : hybris = violence, démesure) est exprimée à travers ces corps qui condensent l'humain et l'animal, corps comiques et carnavalesques, selon les mots de Bakhtine. L'espace de la migration devient soudainement étrange, étranger même, aux yeux des autochtones qui vivent la tropicalisation comme une violence contre leurs normes, contre leurs habitudes. La tropicalisation n'a lieu que dans le choc des cultures. Cette confrontation est soulignée par le narrateur dans le commentaire qui clôt la description 
carnavalesque : « irruption de la Caraïbe des origines; pulsions sauvages de la violence lascive des tropiques, tout cela vibrait sous le regard médusé des archéo-Québécois qui auraient pris panique, n'était la présence massive et rassurante de la flicaille prête à toute éventualité» (Ollivier 39). Au-delà de l'emploi du mot «tropiques » qui est une mise en abyme de la tropicalisation, on retiendra l'aspect conflictuel, agonistique de cette dernière, ce qui rappelle combien il est difficile pour le personnage migrant de transplanter ses valeurs, ses codes dans l'espace réel ou symbolique de l'autre. La tropicalisation est vue comme une menace à l'identité, à l'autochtonie, d'où le regard médusé des « archéo-Québécois », terme employé non sans une certaine ironie pour désigner les Québécois dits de souche, et qui renvoie à un lien presque naturel au sol, à la terre, lien légitime parce que perdu dans la nuit des temps. La présence massive et rassurante des forces de l'ordre, qui a pour effet d'apaiser la crainte de ces autochtones, souligne l'importance de la menace.

Néanmoins, on peut questionner les limites de cette tropicalisation dans Passages. Bien qu'elle soit réelle et non simplement imaginée, désirée, comme dans les Versets Sataniques, elle semble relever d'une certaine irréalité et s'inscrire dans une brève parenthèse temporelle et spatiale; après elle, les choses retournent à la normale, l'autochtonie reprend ses droits. Il s'agirait donc d'une tropicalisation restreinte, contenue dans l'espace et le temps. Elle a lieu sous forme de carnaval, de fête populaire au cours de laquelle les valeurs sont inversées. Elle est également circonscrite symboliquement par les forces de l'ordre, comme on circonscrit un corps étranger pour qu'il n'envahisse ni ne détruise un organisme sain. On pourrait déployer ici toute une série de métaphores médicales pour souligner cette opposition entre I'autochtone et l'allochtone, le pur et l'impur. À la limite, la tropicalisation semble une brève fulgurance, éphémère, presque irréelle. Le romancier a voulu peut-être suggérer que même si le personnage migrant peut réactiver sa culture, il lui est impossible de la relocaliser dans la durée. Au-delà de ces questions, on peut rapprocher la tropicalisation de ce que Homi Bhabha appelle migrant metaphor, ou la migration comme métaphore, c'est-à-dire la production d'une réalité hybride qui peut être diversement interprétée. Ainsi, du point de vue des personnages, le carnaval est vu à la fois comme fête par ceux qui y participent et comme menace par ceux qui le regardent. On est dans le régime de l'ambiguïté, de la double lecture. Mais peu importe son degré de réalité, la tropicalisation est une étape cruciale dans la vie du migrant, elle l'arrache à l'errance, le reterritorialise.

Le désir de tropicaliser l'espace de la migration est exprimé ailleurs de manière métaphorique dans le roman. Normand Malavy, migrant exilé à Montréal, et ses amis sont obsédés par le retour au pays natal. C'est pourquoi les revues que Normand fonde de temps à autres portent toutes des noms qui font référence à l'espace originel. Elles s'appellent Semences, Jonctions, Poteaux. Le dernier numéro d'une de 
ces revues est consacré à une analyse des événements qui précédèrent la chute de la dictature. En se consacrant à l'analyse politique de son pays natal en terre d'accueil, Normand transpose dans l'espace symbolique de ce dernier l'espace sociopolitique de son lieu d'origine. Ces revues parlent d'une réalité autre que celle du pays où elles sont publiées. Leur but est de rassembler une communauté diasporique autour des problèmes qui la concernent.

Dès son arrivée en terre d'exil, le narrateur de Chronique de la dérive douce sait qu'il n'a aucune possibilité de retour: « Je ne suis pas un touriste de passage qui vient voir comment va le monde, comment vont les autres et ce qu'ils font sur la planète. Je suis ici pour rester, que j'aime ça ou pas » (Laferrière 14). Il n'y a pas de tropicalisation de l'espace à proprement parler, mais le narrateur a recours à des stratégies de survie, comme s'il était encore dans le pays natal, car il est conscient des difficultés qui l'attendent en terre d'accueil : « C'est un pays où un chat doit savoir japper s'il veut survivre » (Laferrière 15). Ainsi, de nombreux pigeons du parc Lafontaine finissent dans son plat. Il se promet même de manger des chats, en dépit de la vigilance de la SPCA : «Après les pigeons, viendront les chats, naturellement. Leur viande est souple mais légèrement élastique. À bouillir avec des feuilles de papaye pour attendrir la chair » (Laferrière 31). L'expression « feuilles de papaye », en plus d'être un créolisme la formule correcte serait : feuilles de papayer - semblerait absurde dans ce contexte, puisqu'il n'y a pas de papayer à Montréal. Elle n’a de sens qu'en fonction de la tropicalisation, c'est-à-dire le désir du narrateur de transplanter en pays d'accueil les pratiques de la terre natale.

Ces stratégies, qui rappellent « les pratiques de détour » dont parle Édouard Glissant, semblent relever d'un impératif de survie : on doit s'adapter à son milieu. Formes restreintes de tropicalisation, elles permettent au personnage migrant de se retrouver peu à peu dans un milieu étranger, hostile à la limite. Le migrant s'adapte à l'espace autant que l'espace s'adapte à lui. Ainsi, à la fin du récit, ce qui était étrange, étonnant ne suscite plus la curiosité du narrateur : « À moins d'un mètre de moi. Un long baiser passionné. La fille est en minijupe rouge. Je passe sans m'arrêter » (Laferrière 129). La ville n'est plus étrangère pour le narrateur, car il a cessé de la regarder : « Je ne peux pas dire quand exactement cette ville a cessé d'être pour moi une ville étrangère. Peut-être que j'ai arrêté de la regarder » (Laferrière135). Le narrateur s'est reterritorialisé, c'est-à-dire qu'il n'est plus dans la découverte de l'espace. Au contraire, il semble l'avoir approprié à un point tel qu'il a pris la décision de devenir écrivain.

\section{Conclusion}

http://www.brocku.ca/cfra/voixplurielles05-02/index.html 
On peut distinguer deux phases dans le processus migratoire ou exilique qui déplace le migrant de son espace natal vers une terre étrangère : la première est celle de la perte des repères dans laquelle le migrant doit affronter les non-lieux et faire l'expérience de la déterritorialisation; la seconde est celle de la reterritorialisation, rendue possible grâce à des pratiques de tropicalisation. Néanmoins, ces deux phases ne sont pas tranchées et la transition de l'une à l'autre est souvent imperceptible; de même que déterritorialisation et reterritorialisation ne sont jamais des états mais des processus ouverts et complémentaires. Dans les romans retenus, les liens entre non-lieux et déterritorialisation, tropicalisation et reterritorialisation sont assez évidents. On ne peut pas vivre indéfiniment dans un non-lieu, ce serait alors vivre une errance perpétuelle, qui débouche sur la folie et la mort. Les personnages qui réussissent leur migration sont ceux qui ont la capacité d'implanter eux-mêmes leurs repères dans la terre d'accueil tout en entretenant un rapport sain avec la terre natale. La tropicalisation nous aide à habiter à notre manière notre nouvel espace. C'est ce qu'affirme Émile Ollivier dans son article intitulé « La cuisine nomade. D'Ésaü à nos jours » :

Nous, migrants, en nous insérant dans d'autres sites géographiques, sociaux et culturels, nous apportons avec nous, parmi les débris, les fragments et les miettes qui restent accolés à nos bagages, nos odeurs, nos épices, nos condiments. Ces auxiliaires du quotidien nous aident à recoudre notre mémoire éclatée, à grignoter, à dents de souris, le nouvel espace de vie, à le modeler au besoin selon nos valeurs, nos rêves et nos aspirations. (Ollivier 23)

Le migrant doit se créer sa place dans l'espace de la migration, non pas en brûlant ses vaisseaux, mais en réaménageant sa culture dans ce nouvel espace, en la relocalisant. Ce n'est jamais une opération facile, mais il lui faut savoir utiliser les ruses, « les arts de faire » afin de négocier cette place, entre la mémoire et le présent, entre ailleurs et ici. C'est à ce prix qu'il échappera au deuil inachevé: celui du pays perdu.

\section{Références}

Appadurai, Arjun. Modernity at Large. Cultural Dimensions of Globalization. Minneapolis : University of Minnesota Press, 1998.

Après le colonialisme. Paris : Payot, 2001.

Augé, Marc. Non-lieux. Introduction à une anthropologie de la surmodernité. Paris : Seuil, 1992.

Bhabha, Homi. « Culture's In-Between » in Questions of Cultural Identity, dir. Stuart

Hall et Paul Du Gay. London/New Delhi : Thousands Oaks, Sage Publications, 1996 : 53-60.

Bertrand, Jean-Pierre. «Territorialisation » in Vocabulaire des études francophones. Les concepts de base, dir. Lise Gauvin et Michel Beniamino. Limoges : Presses Universitaires de Limoges, 2005 : 175177.

Baudelaire, Charles. Petits Poèmes en prose (Le Spleen de Paris). Paris : Gallimard, 1973. 
Deleuze/Guattari. Kafka. Pour une littérature mineure. Paris : Les Éditions de Minuit, 1975.

. Capitalisme et schizophrénie. Mille Plateaux. Paris : Les Éditions de Minuit, 1980.

Glissant, Édouard. Le discours antillais. Paris : Gallimard, 1997.

Harel, Simon. Les passages obligés de l'écriture migrante. Montréal : XYZ, 2005.

Kundera, Milan. Les testaments trahis. Paris : Gallimard, 1993.

Laferrière, Dany. Chronique de la dérive douce. Montréal : VLB éditeur, 1994.

Nepveu, Pierre. L'écologie du réel. Montréal : Boréal, 1988.

Ollivier, Émile. Passages. Paris : Le Serpent à Plumes, 1994. «La cuisine nomade. D'Ésaü à nos jours». Vice versa, no 15, 1986, p. 22.

' Les notions de déterritorialisation et de reterritorialisation, qui font maintenant partie de I'appareil théorique de la francophonie, sont définies de la manière suivante dans Vocabulaire des études francophones. Les concepts de base (Limoges, PUL, 2005) : « [L]a déterritorialisation indique un sentiment de perte ou de dépossession, tandis que la reterritorialisation est le processus compensatoire par lequel le sentiment identitaire retrouve un sens » (Bertrand 175).

Pour Deleuze et Guattari, dans le contexte de L'Anti-CFdipe, « se déterritorialiser, c'est quitter une habitude, une sédentarité »(p. 162). On peut donc définir la reterritorialisation comme une sédentarité retrouvée.

J'emploierai ces deux notions dans un sens spatial : la déterritorialisation est le processus par lequel un corps vivant, un système symbolique est arraché à son lieu naturel, natal; par la reterritorialisation, ce corps, ce système est transplanté dans un lieu autre que son lieu naturel, natal.

ii Si cette dimension autobiographique est explicite dans le récit de Laferrière, elle l'est moins dans le roman d'Ollivier. Les deux premières phrases de la quatrième de couverture de Chronique de la dérive douce en soulignent clairement le côté autobiographique : « Voilà trois cent soixante-cinq petites proses comme autant de jours que peut en contenir une année - où l'auteur raconte sa vie quotidienne à l'époque où il n'était qu'un métèque parmi tant d'autres débarqués à Montréal. Dany Laferrière doit se débrouiller avec le peu qu'il retire des dix mille petits métiers offerts à l'immigrant. » Dans Passages, c'est plutôt chez le personnage Normand Malavy qu'on retrouve cette dimension. Il souffre d'insuffisance rénale comme le romancier.

iii Simon Harel parle plutôt «d'habitabilité psychique » pour désigner la possibilité du sujet migrant d'habiter des systèmes symboliques comme l'écriture, la langue. Les passages obligés de l'écriture migrante (Montréal, XYZ, 2005), p. 125. 